\title{
ERRATUM
}

Patrick Salcher • Christoph Adam

\section{Erratum to: Modeling of dynamic train-bridge interaction in high-speed railways}

Published online: 28 August 2015

(C) Springer-Verlag Wien 2015

Erratum to: Acta Mech 226, 2473-2495 (2015)

DOI 10.1007/s00707-015-1314-6

In the original publication of the article, Eqs. (66) and (67) are incorrect. The correct versions of these equations are given below:

$$
\begin{aligned}
& {\left[\mathbf{S}_{1}: \mathbf{J}_{V}\right]=\left[\begin{array}{c:cc}
\mathbf{0}^{\{15 \times N\}} & \mathbf{I}_{V}^{*\{15 \times 15\}} & \mathbf{0}^{\{15 \times 4\}} \\
\mathbf{0}^{\{1 \times N\}} & & \\
\left(\left(\mathbf{N}_{z}^{e, R}\left(\xi_{1}\right)+\mathbf{N}_{z}^{e, L}\left(\xi_{1}\right)\right) \boldsymbol{\Phi}\right) X_{1}(t) / 2 & \\
\left(\left(\mathbf{N}_{z}^{e, R}\left(\xi_{1}\right)-\mathbf{N}_{z}^{e, L}\left(\xi_{1}\right)\right) \boldsymbol{\Phi}\right) X_{1}(t) /(2 b) & \\
\vdots & \mathbf{0}^{\{12 \times 15\}} & \mathbf{G} \\
\mathbf{0}^{\{1 \times N\}} & \\
\left(\left(\mathbf{N}_{z}^{e, R}\left(\xi_{4}\right)+\mathbf{N}_{z}^{e, L}\left(\xi_{4}\right)\right) \boldsymbol{\Phi}\right) X_{4}(t) / 2 & \\
\left(\left(\mathbf{N}_{z}^{e, R}\left(\xi_{4}\right)-\mathbf{N}_{z}^{e, L}\left(\xi_{4}\right)\right) \boldsymbol{\Phi}\right) X_{4}(t) /(2 b) &
\end{array}\right],} \\
& \mathbf{G}=\operatorname{diag}\left[\mathbf{g}_{1}, \mathbf{g}_{2}, \mathbf{g}_{3}, \mathbf{g}_{4}\right], \quad \mathbf{g}_{i}=\left\{\begin{array}{l}
1 \\
0 \\
0
\end{array}\right\}
\end{aligned}
$$

The online version of the original article can be found under doi:10.1007/s00707-015-1314-6.

P. Salcher · C. Adam $(\varangle)$

Unit of Applied Mechanics, University of Innsbruck, Technikerstr. 13, 6020 Innsbruck, Austria

E-mail: christoph.adam@uibk.ac.at

Tel.: +43-(0)512507 61600

P. Salcher

E-mail: patrick.salcher@uibk.ac.at 